\title{
A Brief Scientific Biography of Prof. Alan J. Hunt
}

Alan Hunt was a professor in the Department of Biomedical Engineering at the University of Michigan, and an Associate Editor of Cellular and Molecular Bioengineering, until his death on October 28, 2012. Here we give a brief account of Alan's scientific career, from his doctoral studies at the U. of Washington with Joe Howard, through his postdoctoral work at the U. of Colorado with Dick McIntosh, and finally his faculty research at the U. of Michigan, part of which was conducted in collaboration with David Odde (U. of Minnesota).

Alan Hunt joined Jonathon Howard's lab at the University of Washington shortly after it was set up in the early 1990s; he was Howard's first PhD student. To describe Alan's work in his lab, Howard wrote the following:

"Alan was recruited into the Physiology and Biophysics (PBIO) Graduate Program, and was partially supported by a competitive $\mathrm{PhD}$ fellowship from the Molecular and Cellular Biology Training Program funded by the NIH. Alan graduated from the program in 1993, in almost record time. His project was to measure the force generated by the motor protein kinesin. Motor proteins are molecular machines that convert chemical energy into mechanical work. Kine$\sin$ is a type of motor protein that is distantly related to the myosins, which drive muscle contraction. Kinesins move organelles such as mitochondria and vesicles from one part of a cell to another. This is particularly important in nerve cells where the distances can be so great that diffusion of organelles would require thousands of years for their effective transport in the cell; for example, neurons in the sciatic nerve, which spans from the spinal cord to the foot, are up to one meter long and are dependent upon kinesins to bring materials from where they are made in the cell body, located in the spinal cord, to the synapse, located in the foot.

Kinesin is a tiny machine. With a dimension less than $10 \mathrm{~nm}$ (10 millionths of a millimeter), it is the smallest of all motor proteins, truly a nanomachine. Its track is the microtubule, a long, tube-like polymer of the protein tubulin. Each microtubule is made of 13 parallel protofilaments (so it is like a highway with 13 lanes), and it has an outer diameter of $25 \mathrm{~nm}$. In nerve cells, the length of a single microtubule is up to $0.1 \mathrm{~mm}$, so transport over long distances is achieved by kinesins switching many times from one microtubule to another, partially overlapping, microtubule.
Kinesins walk along microtubules at a rate of only a few millimeters per hour, taking a number of days to traverse a long nerve fiber. On the molecular level, however, this speed is very fast: kinesin moves about 100 of its own lengths per second in steps of $8 \mathrm{~nm}$, i.e., from one tubulin subunit to the next, consuming one molecule of its fuel, ATP, for each step.

Alan and I wanted to measure the force exerted by a single kinesin molecule in order to understand the energetics of this biological machine. How efficient is the engine? Is a single kinesin molecule strong enough to move a cargo, such as a mitochondrion, which is hundreds of times larger than the motor itself? What is the mechanism of its movement? The challenge of the project was how to couple a force to a single kinesin molecule. Alan did this in an ingenious way. He took advantage of the so-called upside down assay in which kinesin is stuck to a surface and free microtubules are introduced to the solution above the surface. The microtubules diffuse randomly, so some of them encounter the surface and bind to the kinesins that are stuck there; if ATP is present, the kinesins walk along the microtubule causing the microtubule to glide across the surface. Because the microtubules are quite long, at least compared to kinesin, they can be seen under a high-quality microscope. Alan lowered the density of kinesin on the surface so each microtubule, whose typical length in these experiments was about $0.01 \mathrm{~mm}$ (or $10,000 \mathrm{~nm}$ ), was driven by just a single kinesin molecule. He could tell that he was in "singlemolecule conditions" because the microtubules swiveled about a single point on the surface where the kinesin was presumably located. This observation indicated that kinesins are very flexible, and Alan used the swiveling to measure kinesin's flexibility (Hunt \& Howard, Proceedings of the National Academy of Sciences, USA, 90:11653-11657, 1993).

To measure the motor force, Alan increased the viscosity of the solution through which the microtubules were moving up to 200 -fold. The idea is that just like moving a spoon in honey, there is a resistive force on the microtubule that increases in proportion to the speed of movement. This resistive force acts as a load on the kinesin molecule and is expected to slow it down. The longer the microtubule, the higher the load, and in this way a full "force-velocity" curve could be mapped out, as shown in Fig. 1. From these data, Alan extrapolated to a maximum force of $4-5 \mathrm{pN}$ for a 

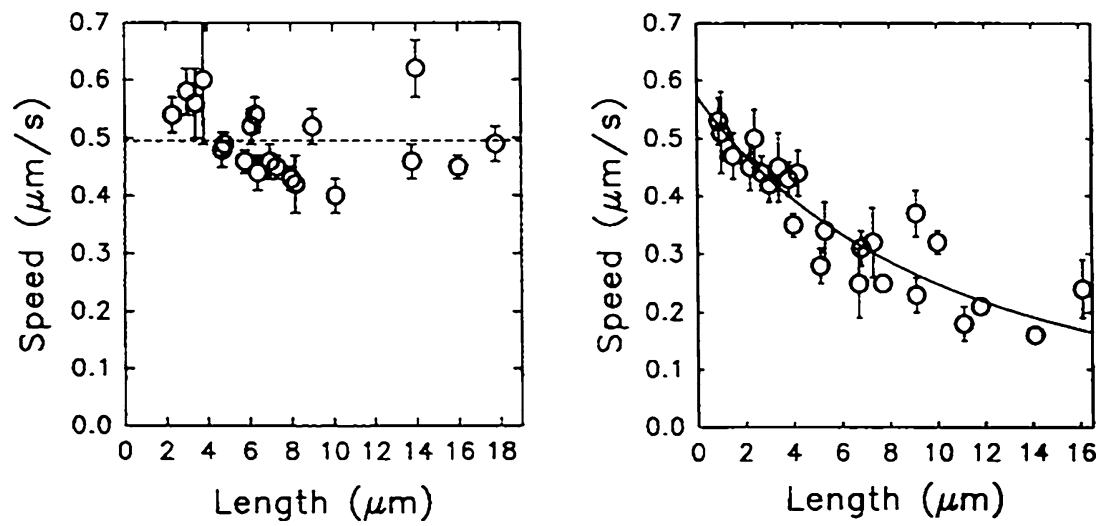

FIGURE 1. Figure from Alan's 1994 paper in the Biophysical Journal showing that as the microtubule length increased, the speed decreased. Alan showed that this was due to the viscous load on the microtubule from the surrounding fluid and estimated that the single-motor force is 4-5 pN, a trillion times smaller than the weight of a pound of flour and equal to the weight of a single red blood cell.

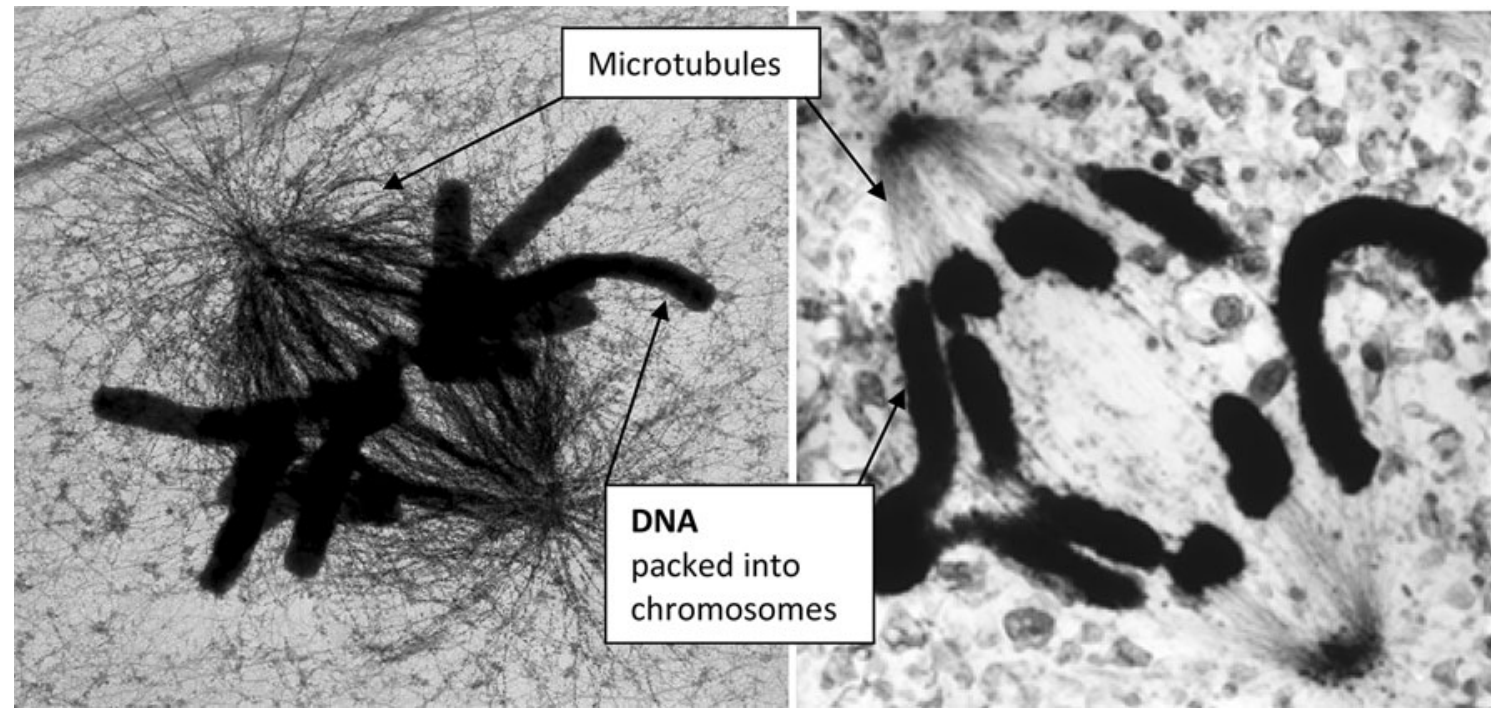

FIGURE 2. Two animal cells in mitosis. On the left, all the chromosomes (dark, sausage-like objects) are already organized at the midplane of the microtubule-based "mitotic spindle", which will pull them apart. On right, the chromosomes are in the process of separating. These pictures of dividing cells were taken with an electron microscope, so the chromosomes look much bigger than they do when seen in a light microscope, as shown in Alan's work below. Modified from Mclntosh, Molodtsov, Ataullakhanov (2012) Biophysics of Mitosis. Quart. Rev. Biophys. 45(2):147-207.

single kinesin molecule exerted against a viscous load (Hunt et al., Biophysical Journal, 67:766-781, 1994). This is a classic measurement of the single molecule force. This value is similar to subsequent measurements (from many labs) of kinesin forces against elastic loads. Alan's measurements have many implications regarding the efficiency of kinesin (up to $50 \%$ ) and also for the mechanism by which force is generated."

For his post-doctoral work, Alan went to Richard McIntosh's lab at the University of Colorado in Boulder. McIntosh writes about Alan:

I was very glad to have Alan join our lab because he had already demonstrated that he was smart, skillful, and well-informed about the motor protein, kinesin. My lab had long been interested in "mitosis", a remarkable process that happens just before a cell divides to become two, as shown in Fig. 2. In preparation for this division, a cell duplicates its DNA then compacts it into tiny, sausage-shaped objects called "chromosomes". For cell division to produce two healthy cells, this pool of duplicated DNA must be organized and moved so each of the two cells formed gets exactly one copy of every chromosome, 46 in the cells of our bodies. Mitosis uses microtubules and kinesins to push and pull on the DNA-containing chromosomes, so they become arranged in an orderly 

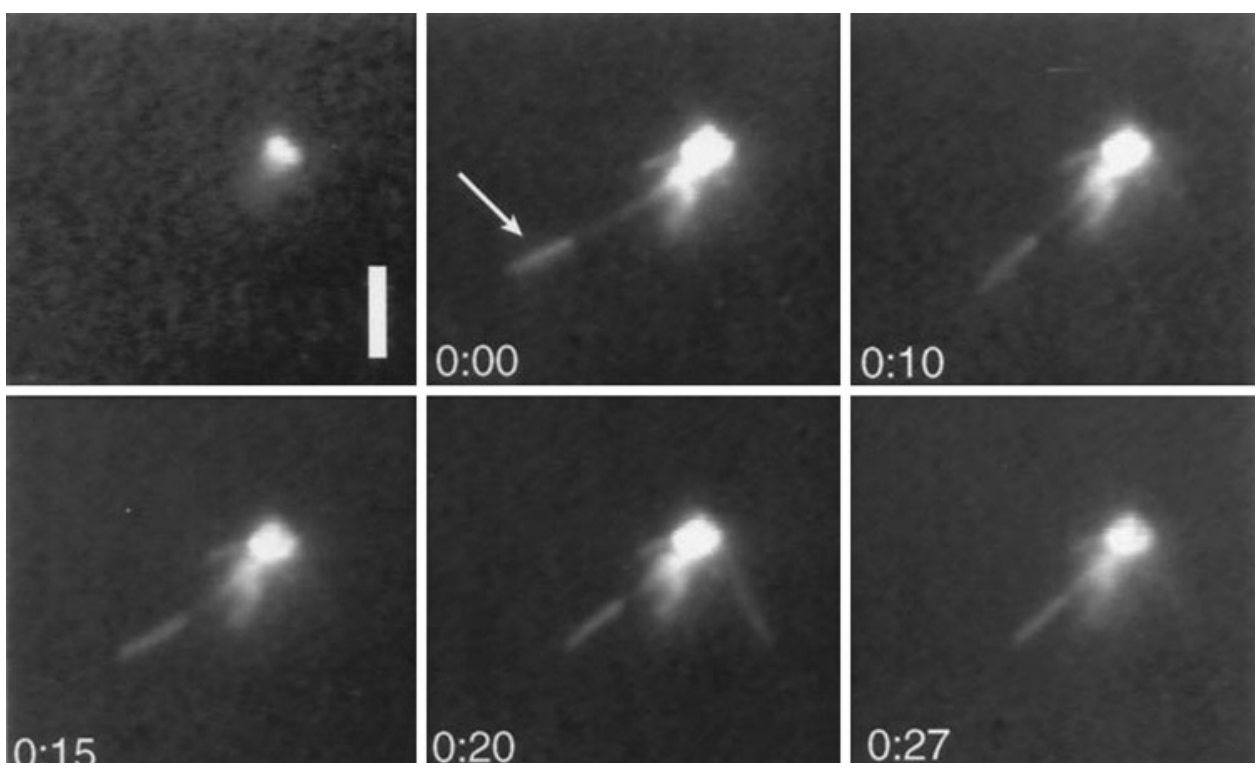

FIGURE 3. These pictures were taken by Alan and published in 1998. The brightest spot in each image is a chromosome. The dim white lines are microtubules, and the arrow points to one end of a microtubule that is brighter than the rest. This is the "minus" end of the microtubule; in a spindle, and it would be at the spindle pole. The microtubule's other end is bound to the chromosome. The numbers are times in seconds. You can see the microtubule shorten while it is attached to the chromosome. Modifed from Hunt and McIntosh, 1998. The Dynamic Behavior of Individual Microtubules Associated with Chromosomes in Vitro. Molecular Biology of the Cell, 9:2857-2871.

structure, called the mitotic spindle, pictured on the left, below. The chromosomes then get pulled apart into two identical sets, one to serve as the genes for each of the two "daughter cells".

In Boulder, Alan studied the interaction between microtubules and chromosomes, which we knew was dependent in part on kinesins. He used a published method to isolate chromosomes from living cells in mitosis, so all the DNA was already duplicated and compacted into chromosomes, as in the images above. He worked out a way to attach the chromosomes to thin pieces of glass, suitable for view in a light microscope. He used a published method to make microtubules that were marked, so he could tell which end was which, when he saw them in the microscope. Now he could watch these microtubules bind to chromosomes and characterize the process, as shown in Fig. 3.

The fascinating thing about what Alan saw was that the microtubules could get longer and shorter while staying bound to a chromosome. The connection they form is fancy in ways we don't yet fully understand, so a chromosome can let microtubules grow and shorten while still holding onto them.

Alan designed a system of lenses that let him bring the beam from a bright infrared laser into the microscope where he was watching the chromosomes and microtubules. With this laser beam, he could grab the microtubules, pull on them, and experiment with the strength of their attachment to the chromosomes.
While in Boulder, Alan also collaborated with two scientists in our university's physics department. We published the fruits of that work in: Mooney, J.F., A.J. Hunt, J.R. McIntosh, and C.T. Rogers (1996) Patterning of functional antibodies and other proteins by photolithography of silane monolayers. Proceedings of the National Academy of Sciences, USA, 93:1228712291. So Alan had a productive time in Boulder, but perhaps more important for his career development, it was also a good time, full of talk about microtubules and kinesins, and how they might work to help cells accomplish their many complicated tasks.

Based on the above accomplishments, Alan earned a job offer from the Dept. of Biomedical Engineering at the University of Michigan, Ann Arbor and a prestigious "Transition grant" from the Burroughs Wellcome Foundation. This gave him financial support to set up the Cellular and Molecular Biomechanics Laboratory. With his graduate student, Ajit Joglekar, Alan published a theoretical paper on the directional instability of chromosome motions in cells (Joglekar, A.P. and A.J. Hunt, A simple, mechanistic model for directional instability during mitotic chromosome movements. Biophysical Journal, 83:42-58 (2002)), and began work with fast lasers, capable of delivering pulses only $10^{-15} \mathrm{~s}$ long. With Gary Brouhard, he developed an advanced laser tweezers system and expanded his earlier work on the interactions between chromosomes and microtubules to characterize the 
(a)

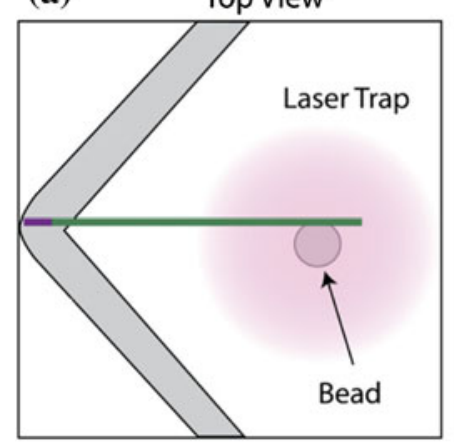

(b)

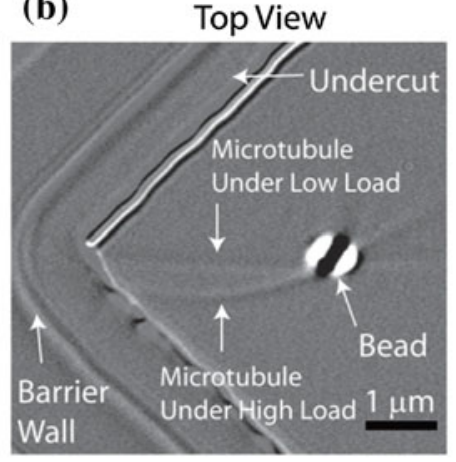

(c)

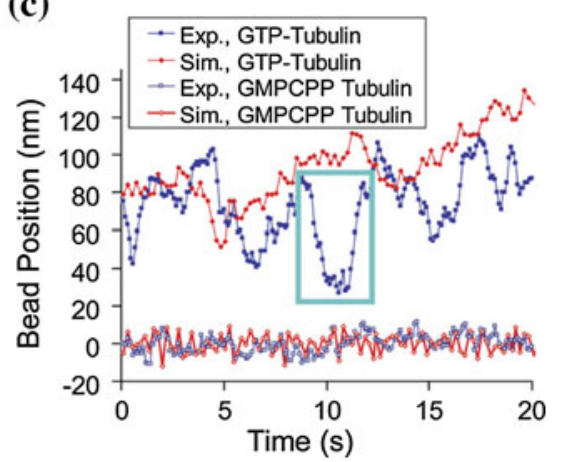

FIGURE 4. Laser-tweezers method for high-resolution tracking of microtubule assembly. (a) schematic, (b) differential-interference contrast image, (c) experimental data (blue) showing large shortening events (e.g., teal box) without catastrophe during periods of overall growth. These dynamics were not detectable in earlier light microscopy measurements, as the laser-tweezers method improved resolution by $\sim 50$-fold spatially $(3.5 \mathrm{~nm})$ and $\sim 10$-fold temporally $(10 \mathrm{~Hz})$. They revealed that microtubules are stabilized by a GTP cap that is much deeper than a single dimer layer $(8 \mathrm{~nm})$, since shortening events often are $>30 \mathrm{~nm}$. Adapted from Schek et al., Current Biology, 2007.

ways in which kinesins bound to the arms of chromosomes can push on the walls of microtubules (Brouhard, G. and A.J. Hunt, Proceedings of the $\mathrm{Na}$ tional Academy of Sciences, USA, 102:13903-13908 (2005)), developing forces that probably help to move the chromosomes to the equator of the mitotic spindles, as shown in the first image above in Fig. 2 (left).

In Ann Arbor, Alan developed new high-resolution methods for studying microtubule dynamics. Odde writes about his collaboration with the Hunt group:

"Alan's previous work focused largely on the forces exerted on and by microtubules. However, at the University of Michigan, he started a new project to revisit an old question: how do microtubules self-assemble? The new twist would be to use laser tweezers and laser-based tracking to measure assembly dynamics at the nanometer scale, nearly two orders of magnitude higher resolution than had been used in the preceding $20+$ years. Understanding these detailed nanomechanics of tubulin addition and loss is vital to understanding, for example, how the anticancer drugs taxol and vinblastine work to alter microtubule assembly and interfere with progression through mitosis.

With his graduate student Henry "Trey" Schek, Alan set up a laser-tweezers based assay to measure the nanometer-scale dynamics of microtubule assembly (Schek, H.T., III, M.K. Gardner, J. Cheng, D.J. Odde, and A.J. Hunt, Microtubule assembly dynamics at the nanoscale, Current Biology, 17:1445-1455 (2007)). Using a weak compressive force $(<1 \mathrm{pN})$ applied to a microtubule-attached bead, Alan's group found that dynamic microtubule ends could be held in close proximity to a microfabricated chamber wall with only modest effect on the net assembly rate $(<10 \%$ reduction due to load; see Fig. 4). By implementing a rapid feedback system $(10 \mathrm{~Hz})$, they were able to maintain a weak force-clamp while simultaneously tracking the trapped bead with $\sim 3.5 \mathrm{~nm}$ nanometer accuracy. This technical tour de force allowed the Hunt group to track microtubule dynamics at unprecedented resolution (for comparison, the previous measurements using light microscopy had accuracy of $\sim 150 \mathrm{~nm}$ at $\sim 1 \mathrm{~Hz}$ ). Thus, the new method, integrating microfabrication and optical trapping, allowed Hunt's group to observe tubulin addition-loss kinetics at near-molecular level resolution (for comparison the tubulin subunits are about $8 \mathrm{~nm}$ long). This technique revealed a number of previously unappreciated features. Most importantly, it showed clear and frequent instances of large shortening events $(>30 \mathrm{~nm})$ during long periods of overall growth (Fig. 4c), implying that the cap of GTP-tubulin, which stabilizes the growing end, must be several dimer layers deep. This finding contradicted earlier estimates, based on lower resolution techniques, which asserted the stabilizing cap could be as small as a single layer of GTP-tubulin at the growing tip.

This finding stimulated further collaboration with me and my graduate student Melissa Gardner to reevaluate tubulin on-off kinetics during microtubule self-assembly. Using integrated nanoscale experimentation and Monte Carlo simulation, we found that the variability in assembly, which reflects the extent of addition-loss dynamics, was far larger than could be explained by published kinetic rate constants going back to the 1980s (see Fig. 5). The conclusions were further strengthened by total internal reflection fluorescence microscopy measurements of microtubule assembly made by Melissa Gardner in Jonathon Howard's lab in Dresden. Whereas the previous studies estimated an on-rate of $50 \mathrm{~s}^{-1}$ and off-rate of $10 \mathrm{~s}^{-1}$ 

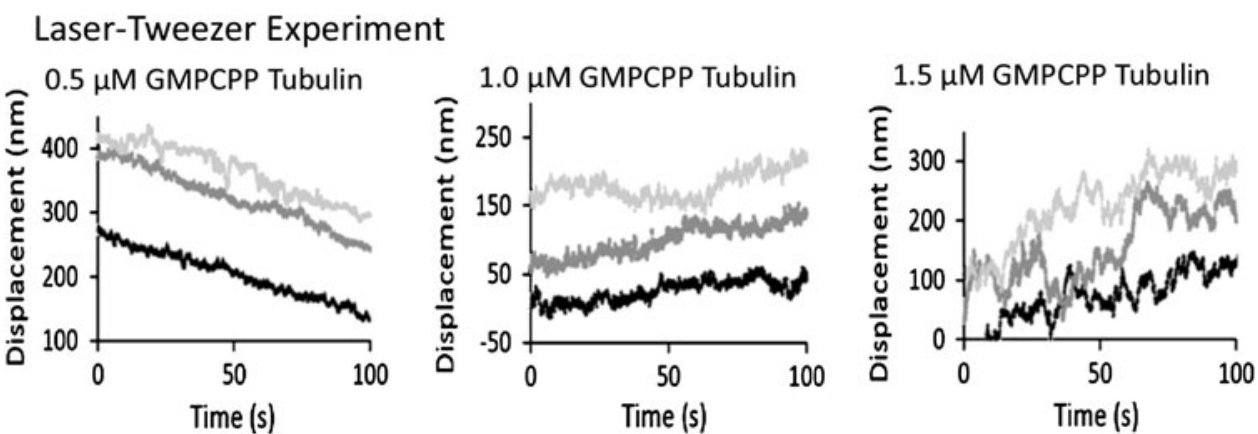

FIGURE 5. Variability of microtubule assembly increases with increasing tubulin concentration. While widely accepted that the subunit addition rate during self-assembly increases with the concentration of the subunits, Alan's group's laser tweezers experiments clearly showed that the off-rate also increases with concentration, as evidenced by the increased variability (and greater frequency of large shortening excursions) at higher concentrations. This finding led to a major revision of how multistranded polymers, such as microtubules, self-assemble. From Gardner et al., Cell 2011.

during microtubule growth under typical conditions (10 $\mu \mathrm{M}$ GTP-tubulin), our estimates were that the onrate is $520 \mathrm{~s}^{-1}$ and off-rate is $480 \mathrm{~s}^{-1}$, so that the net rate was the difference between two very large numbers. This finding implied that even modest alteration of the off-rate would have large consequences for the net rate. This in turn led to the hypothesis that microtubule assembly-regulating proteins and anticancer drugs will strongly influence the assembly process at substoichiometric levels, a concept that we continue to test today, and which we hope will ultimately lead to better cancer therapeutic strategies. The work was published in: Gardner, M.K., B.D. Charlebois, I.M. Janosi, J. Howard, A.J. Hunt, and D.J. Odde, Rapid microtubule self-assembly kinetics, Cell, 146:582-592 (2011).

Beyond his studies of microtubule dynamics, Alan's lab at the U. of Michigan also developed new methods for high-precision machining using femtosecond lasers, which have applications in nanotechnology and can potentially be used to build biomimetic kinetochores. Also, Alan continued to develop a mechanical approach to investigating the mitotic spindle in a collaboration with Duane Compton (Dartmouth College). In addition, Alan worked with stem cell biologists to better understand the mechanics of stem cell division, which has fundamental implications for regenerative medicine and cell-based therapies, a collaboration with Yukiko Yamashita (U. of Michigan). Overall, Alan's scientific body of work was amazingly wide and deep."
Howard, McIntosh, and Odde summarized Alan's scientific career as follows:

"Overall, Alan's career was marked by intense scientific curiosity, open-mindedness, technical depth, and intellectual integrity. Perhaps even more impressive was his intellectual breadth, evidenced by the wide spectrum of topics he investigated ranging from molecular motors to nanomachining to stem cell biology. Finally, Alan's true delight in learning was infectious to all those who worked with him over his career, and he inspired each of us to a higher level of investigation. His presence in our community and in our lives will be greatly missed."

\section{JONATHON HOWARD}

Department of Molecular Biophysics and Biochemistry, Yale University, New Haven, CT, USA

\section{J. RichaRd MCInTOSH}

Department of M.C.D. Biology University of Colorado Boulder, CO, USA

DAvid J. OdDE

Department of Biomedical Engineering, University of Minnesota, Minneapolis, MN, USA

Electronic mail: oddex002@umn.edu 